\title{
Integration of $150 \mathrm{Gbps} /$ fiber optical engines based on multicore fibers and 6-channel VCSELs and PDs
}

\author{
Mikko Karppinen*a , Antti Tanskanen ${ }^{\mathrm{a}}$, Veli Heikkinen ${ }^{\mathrm{a}}$, Petri Myöhänen ${ }^{\mathrm{a}}$, Noora Salminen ${ }^{\mathrm{a}}$, Jyrki \\ Ollila $^{\mathrm{a}}$, Olli Tapaninen ${ }^{\mathrm{a}}$, \\ Petter Westbergh ${ }^{\mathrm{b}}$, Johan Gustavsson ${ }^{\mathrm{b}}$, Anders Larsson ${ }^{\mathrm{b}}$, \\ Rashid Safaisini ${ }^{\mathrm{c}}$, Roger King ${ }^{\mathrm{c}}$, \\ Minsu Ko ${ }^{\mathrm{d}}$, Dietmar Kissinger ${ }^{\mathrm{d}}$, Ahmet Çağrı Ulusoy ${ }^{\mathrm{d}}$, \\ Thierry Taunay ${ }^{\mathrm{e}}$, Lalit Bansale, Lars Grüner-Nielsen ${ }^{\mathrm{f}}$, \\ Efstratios Kehayas ${ }^{\mathrm{g}}$, James Edmunds ${ }^{\mathrm{g}}$, Leontios Stampoulidis ${ }^{\mathrm{g}}$ \\ ${ }^{a}$ VTT Technical Research Centre of Finland, Kaitovayla 1, FI-90571 Oulu, Finland; \\ ${ }^{\mathrm{b}}$ Chalmers University of Technology, Gothenburg, Sweden; \\ 'Philips GmbH ULM Photonics, Ulm, Germany; \\ ${ }^{\mathrm{d}} \mathrm{IHP} \mathrm{GmbH}$, Frankfurt (Oder), Germany; \\ 'OFS Labs, Somerset, NJ, USA; ${ }^{\mathrm{f}} \mathrm{OFS}$, Brøndby, Denmark; \\ ${ }^{g}$ Gooch and Housego, Torquay, United Kingdom
}

\begin{abstract}
Multicore fiber enables a parallel optic data link with a single optical fiber, thus providing an attractive way to increase the total throughput and the integration density of the interconnections. We study and present photonics integration technologies and optical coupling approaches for multicore transmitter and receiver subassemblies. Such optical engines are implemented and characterized using multimode 6-core fibers and multicore-optimized active devices: 850-nm VCSEL and PD arrays with circular layout and multi-channel driver and receiver ICs. They are developed for bit-rates of $25 \mathrm{Gbps} / \mathrm{channel}$ and beyond, i.e. $>150 \mathrm{Gbps}$ per fiber, and also optimized for ruggedized transceivers with extended operation temperature range, for harsh environment applications, including space.
\end{abstract}

Keywords: optical interconnect, parallel optic link, multicore fiber, fiber optics, VCSEL, micro-optics, aerospace electronics

\section{INTRODUCTION}

Multimode optical fiber links are a mature technology for low-power very-short-reach interconnects transferring massive amounts of data between boxes, boards, modules and ASICs in the high-performance computing and communications systems. The multimode transceivers are typically based on 850-nm vertical cavity surface emitting laser (VCSEL) and photodiode (PD) technology. The ever-increasing data rate, interconnection density and capacity requirements are answered both by increasing the VCSEL modulation bandwidths and by increasing the channel counts, i.e. using parallel optic links based on VCSEL arrays.

Typically parallel optic links are implemented using fiber ribbon cables, in which the channel pitch is typically the standard $250 \mu \mathrm{m}$ and the capacity is increased by increasing the number of fibers. This also makes the cable thicker and thus there is a practical limit in increasing the channel count. An alternative solution is the novel multicore fiber (MCF) which enables multiple parallel channels in a single fiber, thus also multiplying the interconnection density. In particular, solid silica-based MCF consisting of several graded-index multimode cores can be compatible with the 850-nm VCSEL

*mikko.karppinen@vtt.fi; phone +358 20722 2293; www.vtt.fi

Optical Interconnects XVI, edited by Henning Schröder, Ray T. Chen, Proc. of SPIE Vol. 9753, 97530S

(c) 2016 SPIE · CCC code: 0277-786X/16/\$18 - doi: 10.1117/12.2214555 
transceiver technology ${ }^{[1]-[3]}$, including also many of its well-known benefits especially related to the cost efficiency. An example of such a fiber by OFS is shown in Figure 1. Even larger throughput densities may be envisaged by fabricating ribbon cables of the multicore fibers. On the other hand, the MCF links are attractive for applications where ribbon cables and connectors are inconvenient. An example is the aerospace application, such as intra-satellite communications, where ribbon connectors are not matured yet and where robust hermetic packaging is often required. A robust hermetic feedthrough applicable for very wide temperature range is easier to implement with a single fiber than with fiber ribbon.
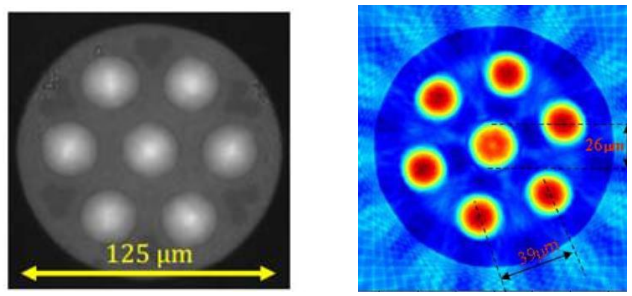

Figure 1. Cross-section of 7-core MM fiber: left) micrograph; right) refractive index profile (by OFS). ${ }^{[2]}$

We investigated the implementation of transmitter and receiver subassemblies based on multicore fibers coupled to VCSEL and PD arrays. These optical engines are developed within a European research project MERLIN ${ }^{[4]}$ which aims to multi-gigabit optical connectivity in the future telecom satellites, which will include on-board processor systems routing data at rates up to several terabits per second between the numerous satellite communication channels. The harsh operation environment also indicates challenging reliability and robustness requirements. Our target is to push to bandwidth density product by combining $25 \mathrm{~Gb} / \mathrm{s}$ (or higher) channel rate and the multicore fiber while enabling very low power dissipation and high reliability in the harsh environment. The packaging is optimized for excellent thermal management enabling reliable high-temperature operation. This makes them attractive also, for instance, for the data center environments where the cooling is a major technical challenge and cost factor.

This paper presents some of the first results of the project with main focus on the design and implementation of the transceiver optical subassemblies and their integration aspects.

\section{TRANSCEIVER TECHNOLOGY}

The developed optical engines, i.e. transmitter and receiver subassemblies (T/ROSA), are targeted for ruggedized midboard transceiver modules, as illustrated in Figure 2. The optical engines are based on $850 \mathrm{~nm}$ VCSEL and PD array technology. Over $150 \mathrm{Gbps}$ full-duplex operation is implemented by communicating through the two 6-core fibers, one for transmission and the other for receiving, and each core transmitting at least at $25 \mathrm{Gbps}$ rate.

The T/ROSA integration approach and the module packaging were optimized to enable surface-mountable module with the $>25 \mathrm{~Gb} / \mathrm{s} / \mathrm{channel}$ electronic input/outputs to the board. These had to be combined with the harsh environment requirement specifications: The transceiver should provide long lifetime ( $>15$ years) with storage temperature range of $55 \ldots+125^{\circ} \mathrm{C}$ and with operation ambient temperature range $-40 \ldots+85^{\circ} \mathrm{C}$ and preferably up to $+100{ }^{\circ} \mathrm{C}$. For both reliability and low power dissipation reasons, the wide temperature operation should be achieved without any active cooling and without any micro-controller or other means for controlling the laser modulation with the temperature variations. Therefore, the high temperature operation can be achieved only by optimizing the T/ROSA integration approach and the module packaging for best possible heat flow from the active devices to the module carrier (note that in vacuum environment, convective heat transfer is not applicable). In addition, active devices optimized for high temperature operation are applied.

In addition, the transceiver should be mechanical shock and vibration resistant, radiation resistant, as well as fully hermetically sealed. Earlier VTT has developed and demonstrated ruggedized transceivers based on hermetic metalceramic packaging and fiber feedthroughs by glass soldering. ${ }^{[5]}$ Similar module packaging technology will be used with the MCF-based transceivers too. 


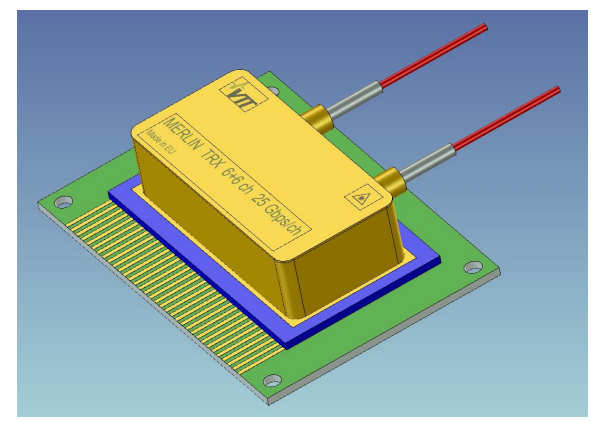

Figure 2. Sketch of hermetically sealed mid-board MCF transceiver module mounted on PCB.

\section{COMPONENT TECHNOLOGIES}

In addition to the multicore fibers, the high-bit-rate high-density multicore optical engines need customized active devices: VCSEL and PD array and multi-channel transceiver ICs. The active devices were made layout compatible for coupling to the selected MCF structure. All devices were also aimed suitable for harsh environment which means for high reliability, long lifetime, radiation resistance, and for operation within wide temperature range.

\subsection{Multicore fibers}

The selected MCF structure is the same as depicted in Figure 1 except that the central core is not included, that is, the fiber has six graded-index multimode cores arranged in circular symmetry with $39 \mu \mathrm{m}$ center-to-center spacing. (Although a MCF can be fabricated to have a seventh core in the center, as shown in Figure 1, it is difficult to make a high-speed VCSEL/PD array with a matching layout.) The fiber outer diameter is the standard $125 \mu \mathrm{m}$, and the fiber has $250 \mu \mathrm{m}$-diameter cladding. The effective diameter of the cores is $26 \mu \mathrm{m}$ and NA is 0.22 . In addition, fiber fan-out assemblies $^{[2]}$ were implemented by OFS to enable separation of the single core signals of the MCF and coupling them to the test equipment with conventional single-core fiber connectors.

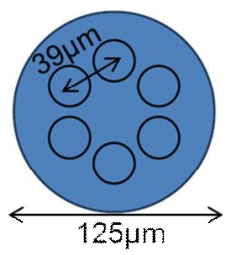

Figure 3. The designed and implemented cross-sectional layout of the 6-core MM fiber.

\subsection{VCSEL arrays}

VCSEL arrays emitting at 850nm were developed and fabricated for the 6-channel MCF links. Similar circular array layout as in the MCF (Figure 3) was selected for the VCSEL emitting apertures, in order to enable e.g. direct butt coupling between the VCSEL array and the MCF cores. The VCSELs were optimized for very high modulation bandwidth well beyond $20 \mathrm{GHz}$ while simultaneously targeting operation temperatures up to $100^{\circ} \mathrm{C}$ or beyond. An example of a 6-channel VCSEL array chip is shown in Figure 4. The technology and results of the early phase fabricated VCSELs were presented in the reference [6] by Westerberg et al.

In the latest generation VCSELs, the beam divergences were reduced for improved coupling efficiency to MCFs. Also the emission spectra were reduced to rms-width of $0.5 \mathrm{~nm}$, enabling higher fiber link bandwidth. The VCSELs exhibit good array uniformity with low threshold currents, high slope efficiencies and $>1 \mathrm{~mW}$ output power up to a temperature of $100^{\circ} \mathrm{C}$. Error-free transmission over standard $50 \mu \mathrm{m}$ core multimode fiber was already demonstrated at 25 Gbit/s/channel rate at $100{ }^{\circ} \mathrm{C}$ and at $40 \mathrm{Gbit} / \mathrm{s} / \mathrm{channel}$ rate at $85^{\circ} \mathrm{C}$. 

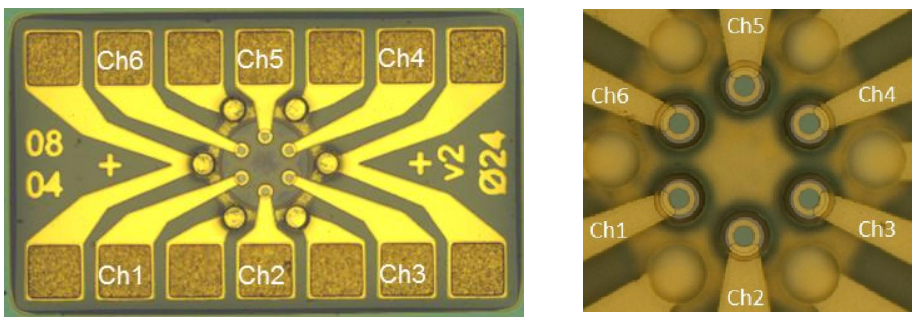

Figure 4. Micrograph of 6-channel MCF-matched VCSEL array: left) complete chip; right) magnification of the mesa area.

\subsection{Photodiode arrays}

For the receivers, high-speed PD arrays were developed in layouts matching to the MCF geometry. The 6-channel GaAsbased PD circular arrays were designed, fabricated and characterized. The design variations included testing of alternative PD active area diameters of 24 and $26 \mu \mathrm{m}$ (Figure 5) but also smaller ones were tested. Large diameter is advantageous in the fiber coupling, but in practice the aperture cannot be further increased due to the small channel spacing. Also typically larger active area results in higher capacitance which leads to smaller bandwidth. PDs with 24 $\mu \mathrm{m}$ apertures were selected for the transceiver implementations. So far they have demonstrated $3 \mathrm{~dB}$ cut-off frequency of $25 \mathrm{GHz}\left(\right.$ at $25^{\circ} \mathrm{C}$ ) and approx. $0.55 \mathrm{~A} / \mathrm{W}$ responsivities at $850 \mathrm{~nm}$.
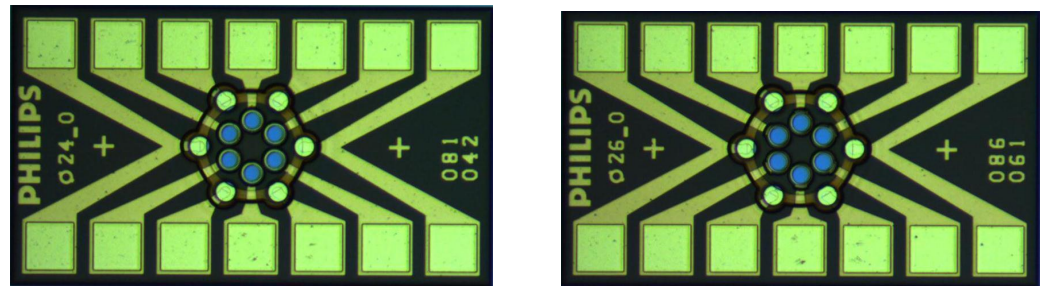

Figure 5. Micrographs of 6-channel circular PD array chips having active area diameters of (left) $24 \mu \mathrm{m}$ and (right) $26 \mu \mathrm{m}$.

\subsection{Multi-channel driver and receiver ICs}

Driver and transimpedance amplifier (TIA) ICs are developed for the MCF-optimized VCSEL and PD arrays (described above). For the optimal optoelectronics integration approach, the monolithic driver and TIA chips were decided to include 3 channels each. This means two driver(/TIA) chips are used in the transmitter(/receiver) with the 6-channel VCSEL(/PD). This enables high integration density and optimal signal integrity with very short bond wires.

The first generation ICs (Figure 6), fabricated in IHP's $0.25 \mu \mathrm{m}$ SiGe BiCMOS process, have already demonstrated clear eye diagrams up to $25 \mathrm{~Gb} / \mathrm{s}$, and the drivers even up to $40 \mathrm{~Gb} / \mathrm{s}$. The driver exhibits a $1.7 \mathrm{~mW} / \mathrm{Gb} / \mathrm{s}$ power efficiency and the TIA circuits exhibits a $2.75 \mathrm{~mW} / \mathrm{Gb} / \mathrm{s}$ at nominal conditions.
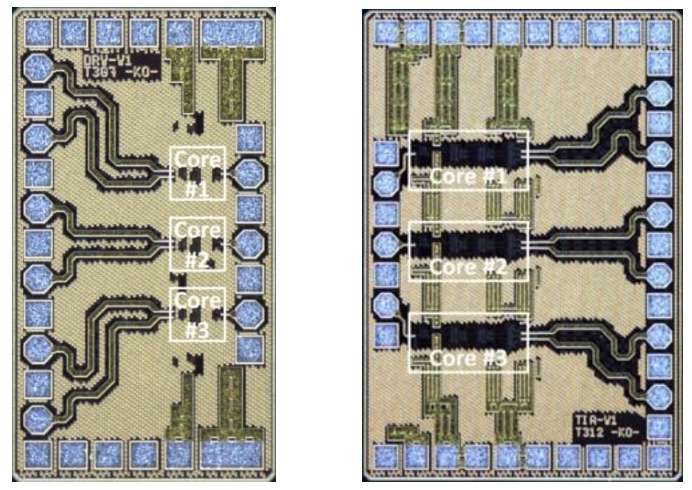

Figure 6. Micrographs of 3-channel Driver IC (left) and 3-channel TIA (right). 


\section{OPTICAL COUPLING}

Optical coupling between the MM MCF and the T/ROSAs was studied by modelling and experiments. In the beginning, alternative coupling schemes were investigated by modelling in order to select and design the module integration approach. With the effective multimode fiber core diameter of $26 \mu \mathrm{m}$, the optical alignment accuracy requirement to a single core is somewhere between the standard single-mode fiber and the standard $50 \mu \mathrm{m}$-core multimode fiber. However, the additional challenges in the optical coupling to the MCF mainly come from the very tight channel spacing, i.e. in our design, the $39 \mu \mathrm{m}$ center-to-center between the adjacent cores (and between the adjacent active areas of the VCSEL/PD arrays). This is much smaller than the $250 \mu \mathrm{m}$ channel pitch of the standard ribbon fiber and it significantly limits the possibilities to exploit optical coupling elements such as lenses. Moreover, the rotation of the MCF about its optical axis has to be controlled precisely during the assembly.

\subsection{Simulation methodology}

The optical coupling design was studied by ray-trace simulations of the optical propagation from VCSEL to PD through the MCF. The simulations were carried out using Zemax optical design software (http://zemax.com). The objectives were to select and optimize the optical design and the integration approach by find out the coupling efficiency between VCSEL/PD array and the MCF, alignment tolerances of the components, back reflection to the VCSEL array, and the optical crosstalk between channels. The simulation model is illustrated in Figure 7. The VCSEL beam shape was defined to the simulation model based on the beam characterization results of the 6-channel VCSELs. The fiber cores were simulated using a graded index profile selected to match with our MCF parameters. The length of the MCF is a trade-off between the simulation accuracy and the simulation time. MCF was chosen to be $3 \mathrm{~mm}$ long, which appeared long enough for stray light to distribute over the MCF and absorb to the MCF primary coating (assumed100\% absorbing).

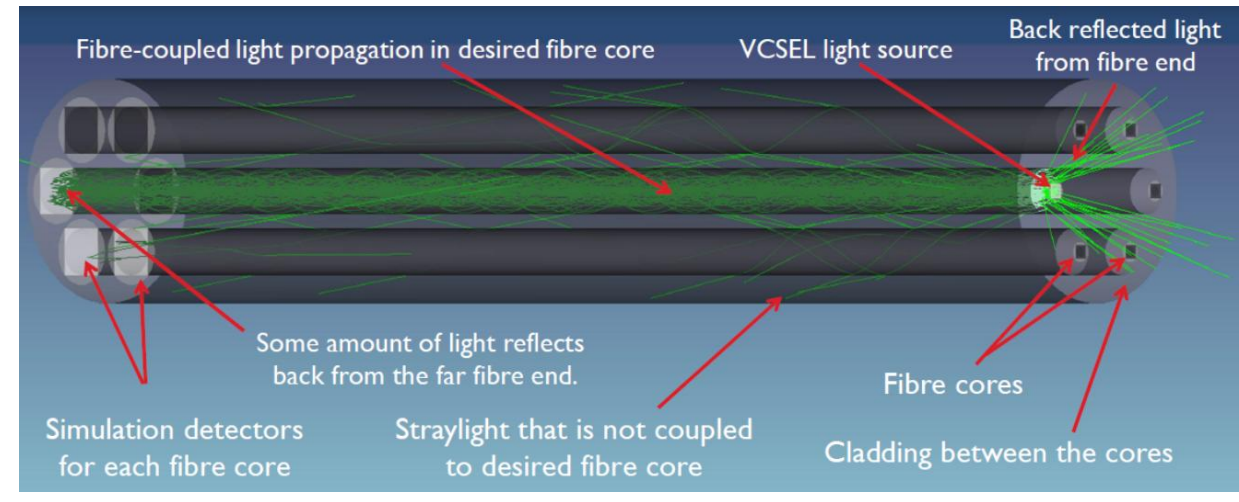

Figure 7. Model for the optical coupling studies by ray-trace simulations of the propagation through the MCF.

\subsection{Coupling scheme}

We studied three alternative optical coupling schemes for the VCSEL/PD-to-MCF couplings: butt coupling, and couplings based on lenses and microlens arrays. The optimized structures are depicted in Figure 8. The key findings are summarized in the following. Note that this comparison of the schemes was simulated with a narrower VCSEL divergence (FWHM $15^{\circ}$ full angle) than in our manufactured latest generation VCSELs (FWHM approx. $25^{\circ}$ full angle) at the maximum driver bias current. Consequently, it somewhat overestimates the coupling efficiency and alignment tolerance values, but still it enables useful comparison. For the butt coupling (Figure 8 a), the simulated VCSEL-to-fiber coupling efficiency was maximum ca $80 \%$ and its transversal alignment (i.e. decenter) tolerances for $1 \mathrm{~dB}$ excess loss were ca $\pm 7 \mu \mathrm{m}$.

Lens-based coupling method (Figure 8 b) applies 1:1 imaging of the VCSEL/PD array to the cores on the MCF end facet. A sufficient coupling was achieved by the use of two aspheric lenses in the telecentric configuration and with the dimensions shown in the Figure. The maximum coupling efficiency from VCSEL to fiber was $77 \%$ (with AR coated lenses). This expanded beam configuration allows a bit relaxed alignment tolerances between the two lenses, but still the 
transversal alignment tolerances of the lenses for $1 \mathrm{~dB}$ excess loss are $\pm 9 \mu \mathrm{m}$. For the fiber and the VCSEL, the $1 \mathrm{~dB}$ lateral alignment tolerances are $\pm 5 \mu \mathrm{m}$. These are rather challenging requirements considering the relatively large dimensions of the structures. These tolerances apply when only a single misalignment is considered at the time. In practice, there are several alignment in the assembly and thus tighter requirements are obviously needed for individual alignments.

Microlens based coupling approach, which is often used with conventional VCSEL arrays, could be sufficiently implemented with the design shown in Figure $8 \mathrm{c}$. It is an etendue matched design using double-sided microlens array. However, the microlens pitch should be smaller and the microlens substrate much thinner $(100 \mu \mathrm{m})$ than of typical microlens arrays. This is needed to achieve sufficient coupling efficiency and to avoid optical cross-talk, i.e. coupling to the adjacent fiber cores or PD active areas. Such a microlens array component is not commercially available off the self, but would require custom development and very advanced manufacturing technology, which are not supported by most vendors. This microlens design enabled maximum coupling efficiency of $90 \%$. The required transversal alignment tolerances for maximum $1 \mathrm{~dB}$ excess coupling loss were $\pm 4 \mu \mathrm{m}$ for each component. (For reference, these values were $83 \%$ and $\pm 2 \mu \mathrm{m}$ when simulated using the measured VCSEL divergence value.)

a)

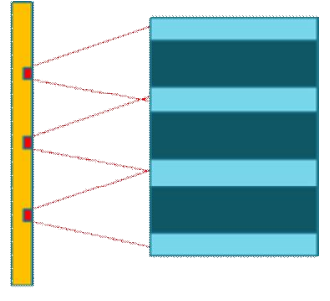

VCSEL/PD Array

$$
\begin{aligned}
& \text { Multicore } \\
& \text { fibre }
\end{aligned}
$$

b)

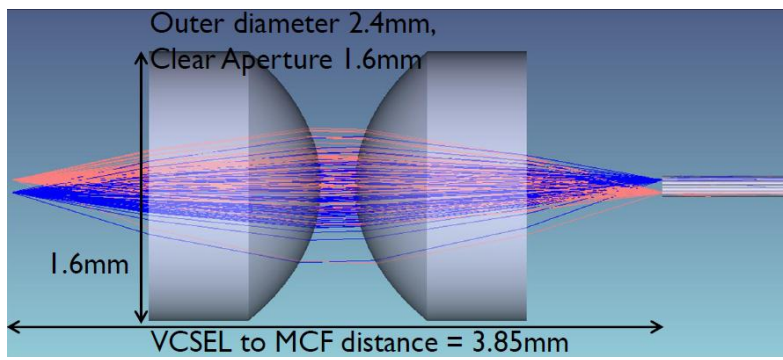

c)
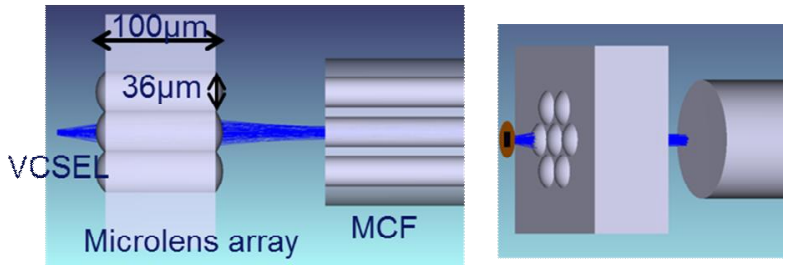

Figure 8. Schematics of the three studied and optimized optical coupling schemes: a) butt coupling, b) lens-based coupling,

c) microlens-based coupling

A comparison of the three coupling approaches is summarized in Table 1. Compared to butt coupling, the microlens scheme enables slightly higher maximum coupling efficiency (in the optimal case) but does not relax the alignment accuracy requirements. The lens based schemes do not show benefits here. However, they would be advantageous if a significant separation between the device and the fiber would be needed, for instance, for a packaging related reason. In our case this was not needed, thus butt coupling was selected as the best choice.

Table 1. Comparison of alternative optical coupling schemes for vertically assembled T/ROSA.

\begin{tabular}{|c|c|c|c|c|c|}
\hline Method & $\begin{array}{l}\text { Coupling } \\
\text { efficiency }\end{array}$ & $\begin{array}{c}\text { Component } \\
\text { availablity }\end{array}$ & $\begin{array}{l}\text { Component } \\
\text { costs }\end{array}$ & $\begin{array}{c}\text { Ease of } \\
\text { assembly }\end{array}$ & $\begin{array}{c}\text { Stars in } \\
\text { total }\end{array}$ \\
\hline Butt coupling & ist & 礼礼 & 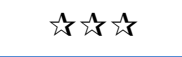 & 动むた & 11 \\
\hline Discrete lenses & ist证 & 礼礼 & 站访 & 虰光 & 9 \\
\hline Microlens arrays & 引认办 & it & th & is & 6 \\
\hline
\end{tabular}

The alternative integration approach is to mount the optoelectronics on the module substrate and bend the light 90 degree when coupling to the fiber. Due to the bending structure the separation between the VCSEL/PD and the MCF cannot be very short. This indicates a need to use some kind of lens optics too, for instance, the mirror could be placed between the 
two lenses in the aforementioned lens based scheme. Several alternative optical coupling schemes including $90^{\circ}$ bend (various combinations of mirrors/prisms and lenses) were studied and modelled too, but none of them was found applicable enough.

\subsection{Optical alignment tolerances}

The simulated alignment tolerances for the butt coupling scheme (using the measured VCSEL divergence with max driver bias) are presented in Figure 9. The figures on the left present the transversal misalignment tolerances for the VCSEL and PD and the figures on the left the tolerances along the optical axis. Figure 9 a was calculated for VCSELfiber separation of $10 \mu \mathrm{m}$. The Figure $9 \mathrm{~b}$ shows that due to the relative high VCSEL beam divergence, the butt coupling approach requires a small separation (ca $20 \mu \mathrm{m}$ or less) between the device and the MCF end facet. The VCSEL divergence and the fiber NA also limit the maximum coupling efficiency to ca $70 \%$. Very short separation is needed also in the receiver end, as shown in Figure 9 d, because the diameter of the fiber core is even a bit larger than the diameter of the PD active area. Due to the same reason the receiver coupling is more sensitive to the transversal misalignments than the transmitter, as shown in Figure $9 \mathrm{c}$, where the PD-fiber separation was assumed $15 \mu \mathrm{m}$.

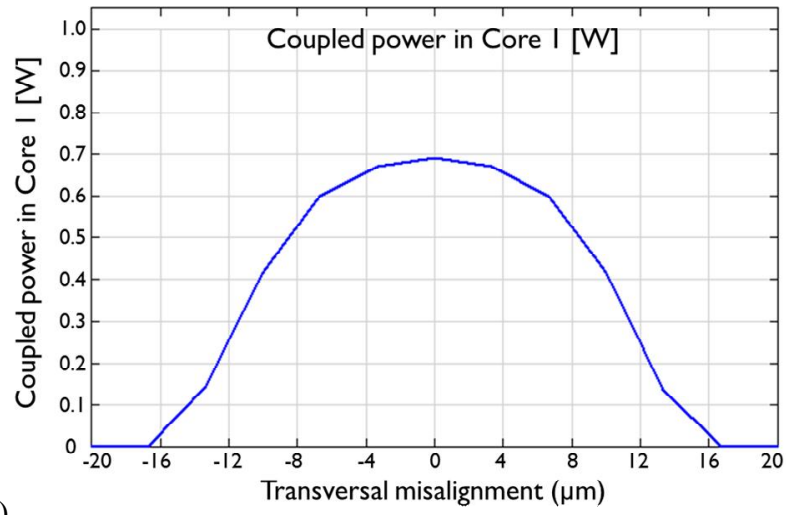

a)

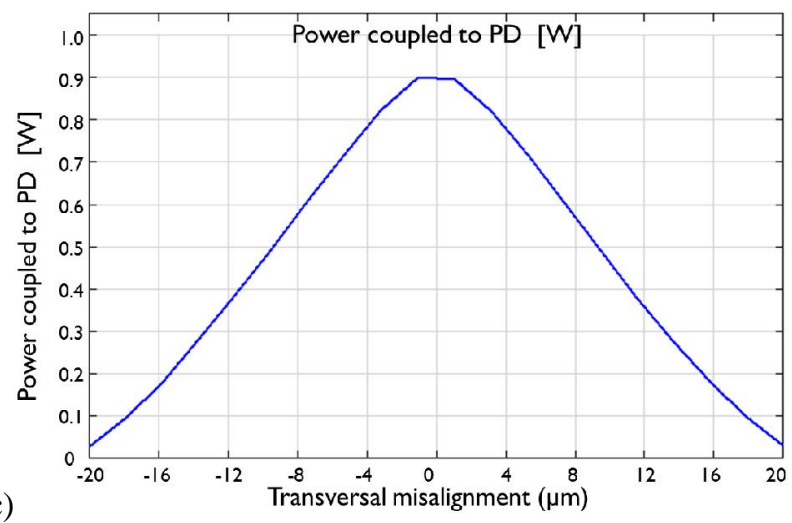

b)
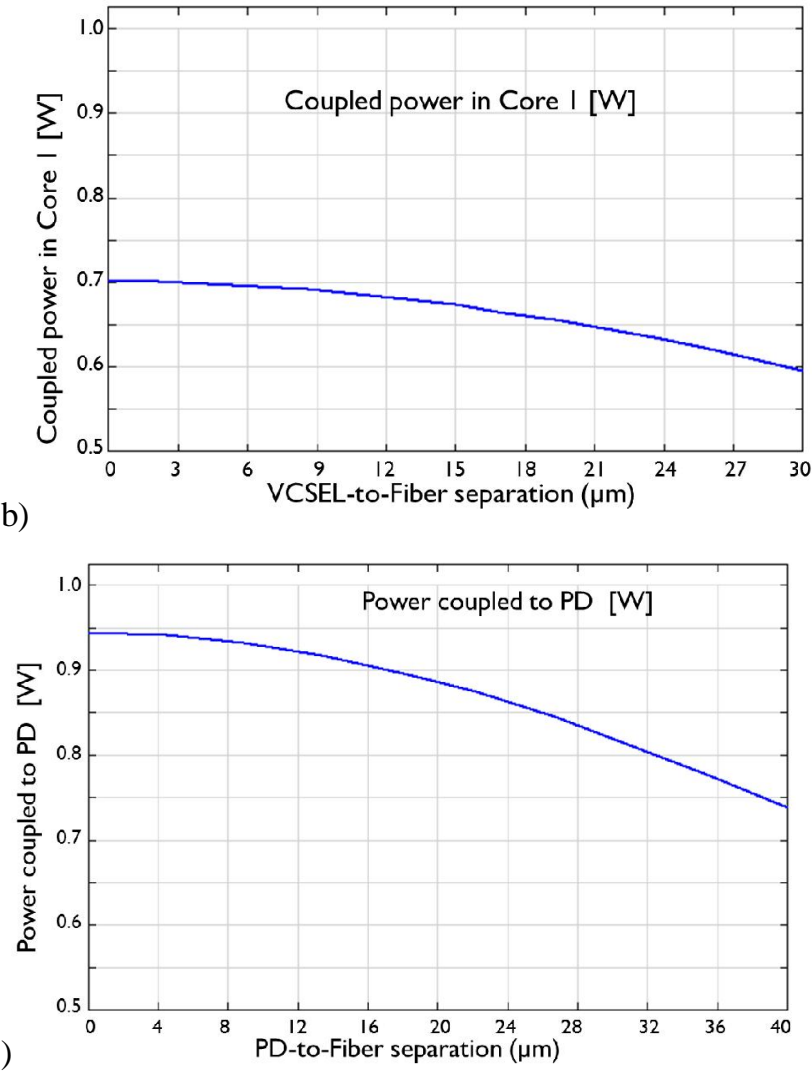

Figure 9. Simulated butt coupling efficiencies versus misalignments both perpendicular and along the optical axis: upper) VCSEL-to-MCF coupling, lower) MCF-to-PD coupling. (Input powers normalized to $1 \mathrm{~W}$.)

Optical butt coupling of the VCSEL array to the MCF was experimentally demonstrated using the fabricated TOSAs (see Chapter 5.3). In addition, the alignment tolerances were investigated experimentally. Figure 10 presents the effect of the transversal misalignment in the VCSEL-to-MCF coupling for two different bias currents. Larger VCSEL current results in larger beam divergence and the $4 \mathrm{~mA}$ bias results in approx. the same divergence as used in the aforementioned simulations. The VCSEL-MCF separation could not be measured accurately but was estimated to be ca 10-15 $\mu \mathrm{m}$. The simulated and measured results are quite similar. The small differences between the shapes of the curves are at least partly due to the fact that the actual VCSEL beam is not fully rotationally symmetric (because multimode). The measurement shows slightly higher sensitivity to misalignments which probably indicates that the VCSEL-MCF 
separation was a bit larger in the experiment than assumed in the simulations. When the separation was increased the coupling was measured to decrease similarly as simulated (Figure $9 \mathrm{~b}$ ).

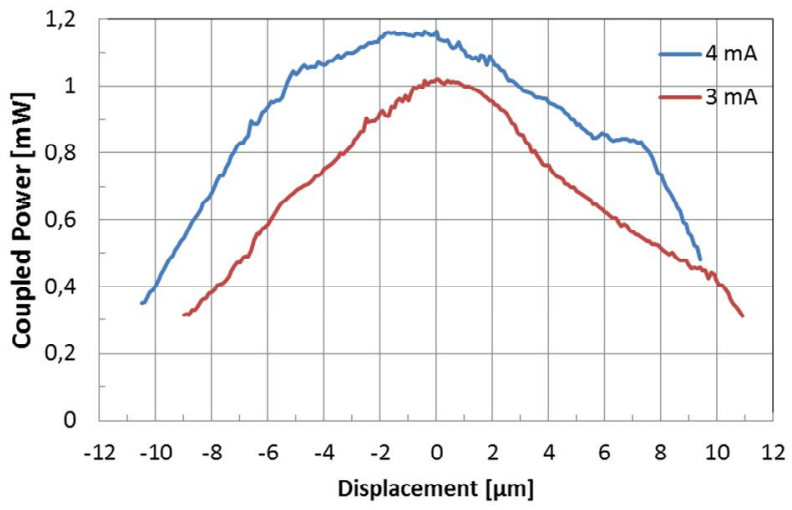

Figure 10. Measured VCSEL-to-MCF coupling versus misalignment perpendicular to the optical axis, at two bias currents.

\subsection{Optical cross-talk}

The optical crosstalk was studied also more carefully by simulations. For the butt coupling case, the unintended coupling from a single VCSEL aperture to the other cores of the MCF is staying below $-32 \mathrm{~dB}$, i.e. negligible in the data link, when the VCSEL-to-fiber separation is $20 \mu \mathrm{m}$ or less. The experiments supported these results too. In the case presented in Figure 10, the crosstalk was at least smaller than $-20 \mathrm{~dB}$ (measurement accuracy). The crosstalk characteristics were also studied in more detail by Westerberg et al. ${ }^{[7]}$

\section{INTEGRATION DESIGN AND FABRICATION OF OPTICAL ENGINES}

Transmitter and receiver optical subassemblies were designed and implemented using the aforementioned components and optical designs and by addressing the MCF transceiver module requirements and targets described in Chapter 2.

\subsection{Fiber coupling and chip integration approach}

Transceiver integration approach and especially the fiber coupling approach are illustrated in Figure 11. The 6-channel VCSEL/PD chip is mounted on the optical subassembly substrate between the two 3-channel driver/receiver ICs. The OSA substrate is vertically mounted on the module substrate. Multicore fiber is butt coupled to VCSEL/PD array. Further, this design enables hermetic fiber feedthroughs to the metal package. The chips are contacted by wire bonding, as shown in Figure 10 right. The chips are mounted into cavities on the T/ROSA substrate as shown in Figure 11 right (but not depicted in the left figure). Thus the bond wire lengths can be kept very short (on the order of $100 \mu \mathrm{m}$ ) resulting in good high-bit-rate signal integrity.
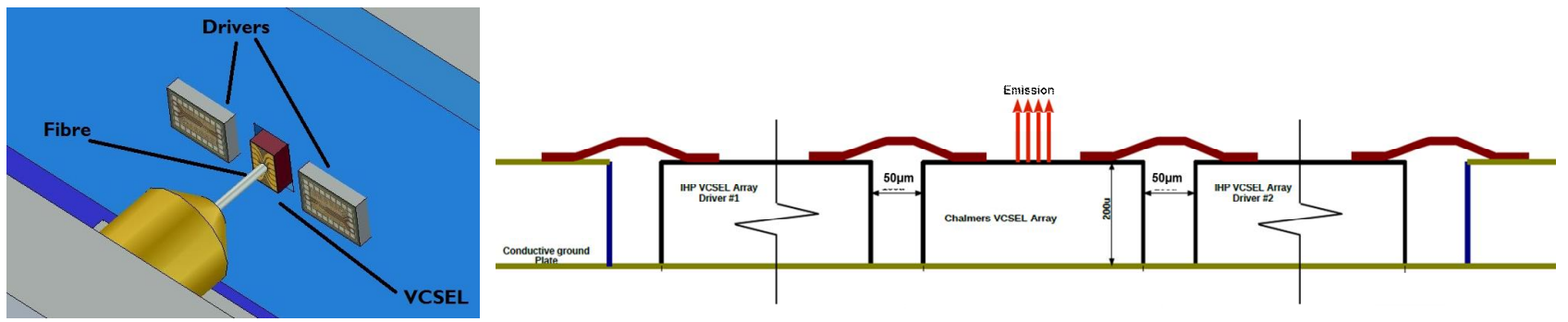

Figure 11. left) Schematics of the integration scheme and fiber coupling approach. Right) Chip and wire bonding scheme. 


\subsection{Thermal management}

The targeted extended operation temperature range, especially the applicability at ambient temperatures up to $100{ }^{\circ} \mathrm{C}$, without any active cooling (see Chapter 2) mean that the thermal management of the packaging structure should be optimized in such a way that the chip temperatures will not raise significantly higher than the ambient environment. In practice, thermal conductivity of all structures, including the OSA and module substrates and the interface between them, should be maximized.

For the optimal thermal management combined with very good high-frequency characteristics, Cu-core circuit boards were chosen as the T/ROSA substrates and LTCC ceramic circuit board as the module substrate. The latter also includes thermal vias and supports hermetic high-reliability packaging. The thermal simulation results illustrated in Figure 12 predicted only circa $3^{\circ} \mathrm{C}$ higher temperature for the VCSEL and only circa $5^{\circ} \mathrm{C}$ higher temperature for the ICs as compared to the mother board (PCB) of the module, respectively. The mother board was assumed connected to a heat sink at ambient temperature.

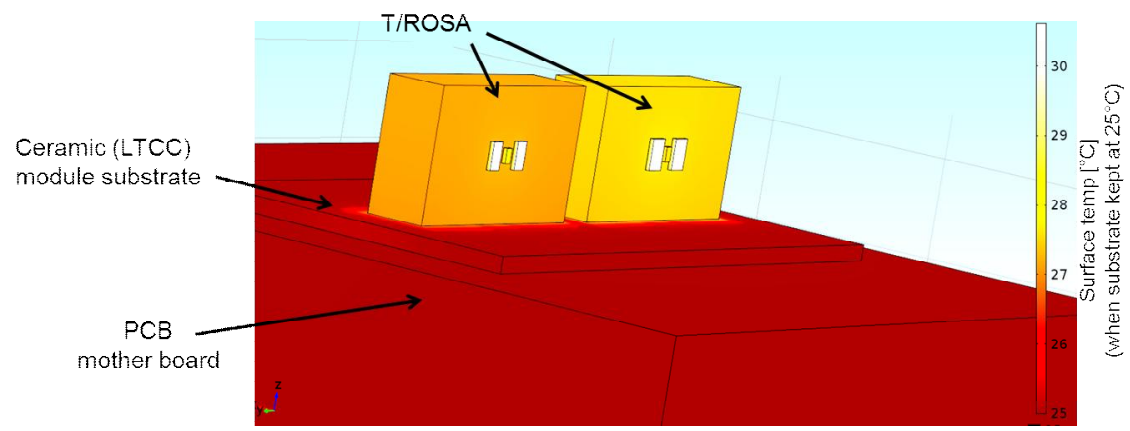

Figure 12. Thermal modelling result of the transceiver module structure, when operational and the mother board is at $25^{\circ} \mathrm{C}$.

\subsection{TOSA and ROSA assembly}

The first multicore optical engines were manufactured for characterization and testing. At first the Cu-core substrates were designed and fabricated, and then the TOSAs and ROSAs were assembled. Micrographs showing the active devices on the subassemblies are presented in Figure 13.
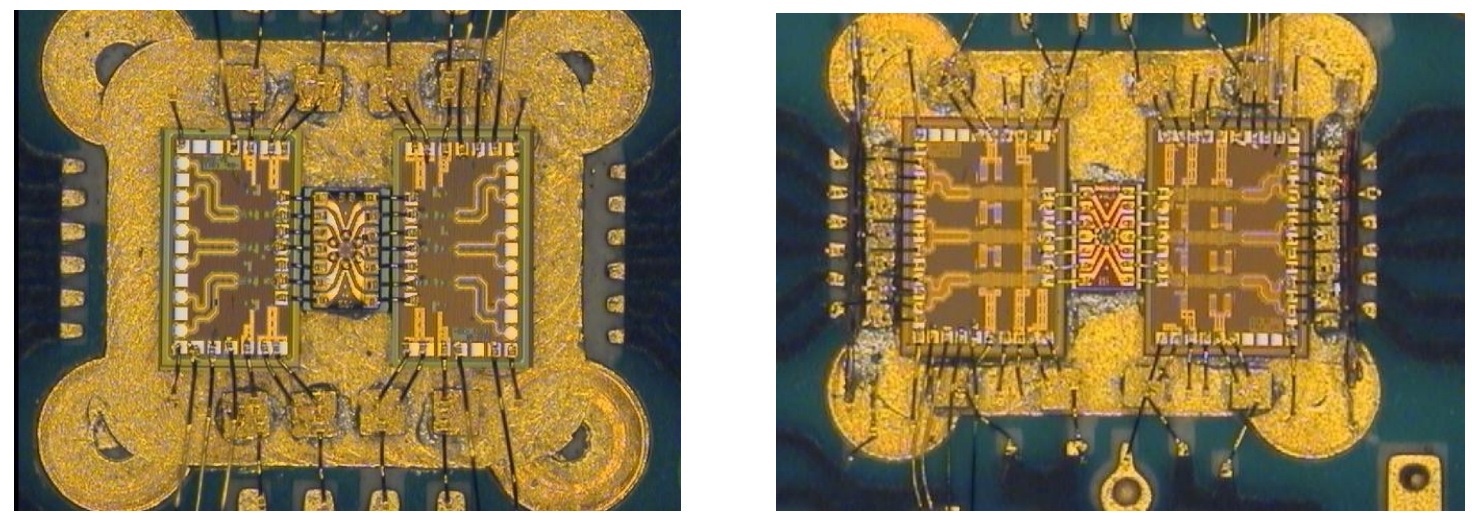

Figure 13. The active devices bonded on the OSA substrates: left) transmitter subassembly, right) receiver subassembly. (Note: The high-speed I/O pads of the driver do not have bond wires, since this TOSA was characterized by probing.)

\section{TESTING OF OPTICAL ENGINES}

The first high-bit-rate transmission experiments with the TOSAs and ROSAs were carried out by single core OM4 type multimode fibers. The TOSA test results are presented in Figure 14, which shows the bit error rate (BER) results as a 
function of the received optical modulation amplitude (OMA) at $10 \mathrm{~Gb} / \mathrm{s}$ and at $25 \mathrm{~Gb} / \mathrm{s}$, at room temperature. The received power was controlled by the use of variable optical attenuator. Also, the eye diagrams are shown at four different bit-rates. It should be noted that these results were partly limited by the performance of the optical receiver used in the experiment. In addition, the driver was operated without the pre-emphasis function.

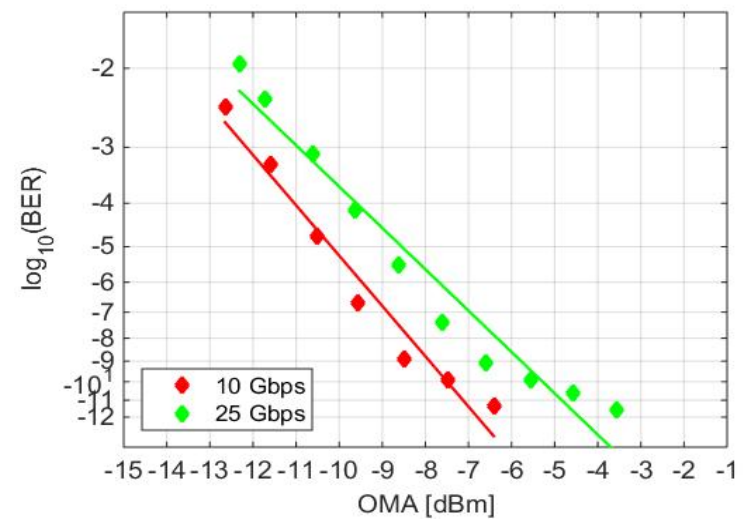

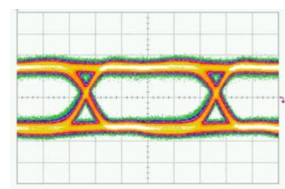

(a) 10 Gbps

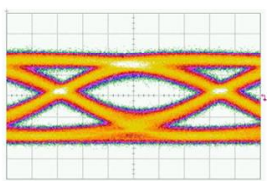

(a) 36Gps

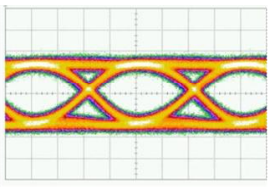

(a) 25Gbps

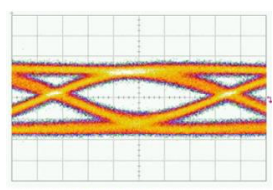

(a) $40 \mathrm{Gbps}$

Figure 14. left) BER of the TOSA as a function of the received OMA. Right) Optical eye diagrams of the TOSA.

\section{CONCLUSIONS}

Optical engines were designed and implemented based on custom developed MM multicore fibers, 850-nm 6-channel VCSEL and PD arrays, and multi-channel driver and TIA ICs, all supporting at least $25 \mathrm{~Gb} / \mathrm{s} / \mathrm{channel}$ operation. The integration technology was optimized for high-reliability transceivers and for extended operation temperature range. The VCSELs have already shown $25 \mathrm{~Gb} / \mathrm{s}$ operation at temperatures up to $100{ }^{\circ} \mathrm{C}$, and the thermal simulations of the designed transceiver module structure indicated only a few degrees temperature difference between the active devices and the ambient environment. Alternative optical coupling methods to the MCF were studied and finally the integration approach based on butt coupling was selected.

Currently, the implementation of complete transceiver modules is ongoing. Also enhanced versions of the active devices are manufactured.

The research is aiming for harsh environment applications, especially for intra-satellite links. However, the technology is expected applicable also for other applications, for instance, datacenters would benefit of the low power dissipation and the reliable operation at high temperatures, which enables low overall cooling costs.

\section{ACKNOWLEDGEMENTS}

The research leading to these results has received funding from the European Union Seventh Framework Programme for research, technological development and demonstration under the Grant number 607274 (MERLIN project). The work at Chalmers was also supported by the Swedish Foundation for Strategic Research. The authors would like to thank the colleagues at the project partner Thales Alenia Space for the guidance and discussion. In addition, we thank a number of colleagues who have contributed the technical work.

\section{REFERENCES}

[1] Rosinski, B. J., Chi, W. D., Grosso, P., and Le Bihan, J., "Multichannel transmission of a multicore fiber coupled with vertical-cavity surface-emitting lasers," J. Lightw. Technol., vol. 17, no. 5, pp. 807-810, 1999. 
[2] Zhu, B., Taunay, T. F., Yan, M. F., Fishteyn, M., Oulundsen, G., and Vaidya, D., "70-Gb/s multicore multimode fiber transmissions for optical data links," IEEE Photonics Technology Letters, vol. 22, no. 22, pp. 1647-1649, Nov 2010.

[3] Lee, B.G., Kuchta, D.M., Doany, F.E., Schow, C.L., Pepeljugoski, P., Baks, C., Taunay, T.F., Zhu, B., Yan, M.F., Oulundsen, G., Vaidya, D.S., Luo, W., and Li, N., "End-to-End Multicore Multimode Fiber Optic Link Operating up to $120 \mathrm{~Gb} / \mathrm{s}$," IEEE J. Lightw. Technol., vol. 30, iss. 6, pp. 886-892, 2012.

[4] Stampoulidis, L., Kehayas, E., Karppinen, M., Tanskanen, A.; Heikkinen, V., Westbergh, P., Gustavsson, J., Larsson, A., Grüner-Nielsen, L., Sotom., M., Venet, N., Ko, M., Micusik, D., Kissinger, D., Ulusoy, A. C., King, R., and Safaisini, R., "The European project "MERLIN" on multi-gigabit, energy-efficient, ruggedized lightwave engines for advanced on-board digital processors," International Conference on Space Optics (ICSO), 7-10 Oct 2014, Tenerife, Canary Islands, Spain. European Space Agency ESA (2014).

[5] Karppinen, M., Heikkinen, V., Juntunen, E., Kautio, K., Ollila, J., Sitomaniemi, A., and Tanskanen, A., "Optical transceivers for interconnections in satellite payloads," Proc. SPIE 8630, Optoelectronic Interconnects XIII, (2013).

[6] Westbergh, P., Gustavsson, J. S., and Larsson, A., "VCSEL Arrays for Multicore Fiber Interconnects with an Aggregate Capacity of 240 Gbit/s," IEEE Photonics Technology Letters, vol. 27, no. 3, Feb 2015.

[7] Westbergh, P., Gustavsson, J. S., Larsson, A., Bansal, L., and Grüner-Nielsen, L., "Crosstalk Characteristics and Performance of VCSEL Array for Multicore Fiber Interconnects," IEEE Journal of Selected Topics in Quantum Electronics, vol. 21, no. 6, Nov/Dec 2015. 